tion of stented ductus was used as a "patch" for arch reconstruction. The arch was reconstructed by sewing the lateral free edges of the divided ductus to the divided native aortic arch. Continuity was then restored with the main pulmonary artery and proximal aorta (Figure 1, F).

The branch pulmonary artery bands were removed, and the branches were dilated. Continuity was restored by folding the pulmonary artery button and sewing the anterior free edges together. A bidirectional cavopulmonary shunt was constructed. The patient's postoperative course was unremarkable, and he was discharged on postoperative day 9. After 1 month, the postoperative echocardiogram demonstrated an unobstructed reconstructed aortic arch.

\section{Discussion}

The use of retained stented ductal material to reconstruct the aortic arch offers several advantages over traditional homograft reconstruction.

1. The stented material is "preformed" to fit the contour of the transverse aortic arch.

2. Continuity between the ductal material and the descending aorta avoids the need for exposure of this area, obviates any distal sewn anastomoses. and thereby prevents bleeding and reduces potential for recurrent laryngeal nerve injury.
3. Avoidance of homograft material may improve the potential for future transplantation. ${ }^{2}$

Potential disadvantages include the following:

1. There is potential for aneurysmal ductal dilation. The retention of the stent deeply embedded in the tissue, however, leaves the tissue "wire-reinforced," and this risk seems small.

2. Any circumferential retention of stent material will likely require reoperation for coarctation; consequently, meticulous removal of the stent in the posterior aorta is important.

3. The growth potential of the isthmus area after stent removal is not known.

The use of retained stented ductal material facilitates the hybrid second-stage procedure and thereby improves the relative merit of the hybrid management strategy in comparison with the Norwood strategy. Long-term follow-up will be required.

\section{References}

1. Akintuerk H, Michel-Behnke I, Valeske K, et al. Stenting of the arterial duct and banding of the pulmonary arteries: basis for combined Norwood stage I and II repair in hypoplastic left heart. Circulation. 2002; 105:1099-103.

2. Meyera SR, Campbell PM, Rutledge JM, et al. Use of an allograft patch in repair of hypoplastic left heart syndrome may complicate future transplantation. Eur J Cardiothorac Surg. 2005;27:554-60.

\title{
Right minithoracotomy as an alternative approach for endovascular repair of thoracic aortic aneurysm
}

\author{
Igor Vendramin, MD, ${ }^{a}$ Gianluca Piccoli, MD, ${ }^{\text {b }}$ Daniele Gasparini, MD, ${ }^{\mathrm{b}}$ and Ugolino Livi, MD, ${ }^{a}$ Udine, Italy
}

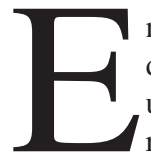

ndovascular repair of chronic descending thoracic aortic diseases (aneurysm and dissection) is now a widely used treatment with satisfactory short- and midterm results in terms of morbidity and mortality. ${ }^{1,2}$ Nevertheless, absence of available traditional access (femoral artery, iliac artery, right subclavian artery) remains a great challenge for

From the Division of Cardiothoracic Surgery, Department of Cardiopulmonary Sciences, ${ }^{\mathrm{a}}$ and the Division of Vascular and Interventional Radiology, Department of Radiologic Sciences, ${ }^{\mathrm{b}}$ University of Udine Medical School, Udine, Italy.

Received for publication June 8, 2007; accepted for publication June 22, 2007.

Address for reprints: Igor Vendramin, MD, Division of Cardiothoracic Surgery, Department of Cardiopulmonary Sciences, Piazzale Santa Maria della Misericordia, Udine 33100, Italy (E-mail: Igor.vendramin@ virgilio.it).

J Thorac Cardiovasc Surg 2007;134:1589-91

$0022-5223 / \$ 32.00$

Copyright $\odot 2007$ by The American Association for Thoracic Surgery doi:10.1016/j.jtcvs.2007.06.038 physicians who have to explore new strategies to avoid major surgical approaches.

We report the case of a thoracoabdominal aortic aneurysm treated with stent grafts implanted through the ascending aorta with a right anterior minithoracotomy approach.

\section{Clinical Summary}

A 59-year-old man had a thoracoabdominal aneurysm and severe atherosclerotic disease of the femoral and iliac arteries and brachiocephalic trunk, causing severe and obstructive stenoses. The abdominal aorta was not available because of the aneurysmatic disease nor was the right subclavian artery owing to kinking. Preoperative imaging (computed tomographic [CT] scan) demonstrated an aneurysm involving the descending aorta between the left subclavian artery (LSA) and the celiac trunk (Figure 1, A) and a second subrenal abdominal aortic aneurysm.

The procedure was performed with the patient under general anesthesia with double-lumen intubation, and guidance was provided by transesophageal echocardiography and fluoroscopy. A 7.0-cm incision was made at the level of the second right anterior intercostal space (Figure 2), and the right internal thoracic artery was dissected and cut. A small rib retractor was used to open the pericardium, and a side-biting clamp was introduced to partially 

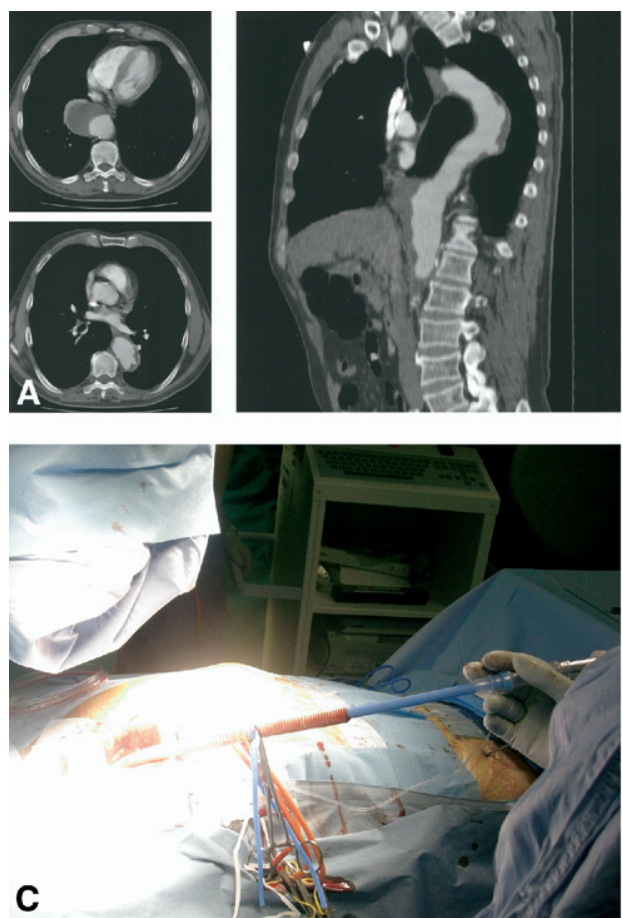
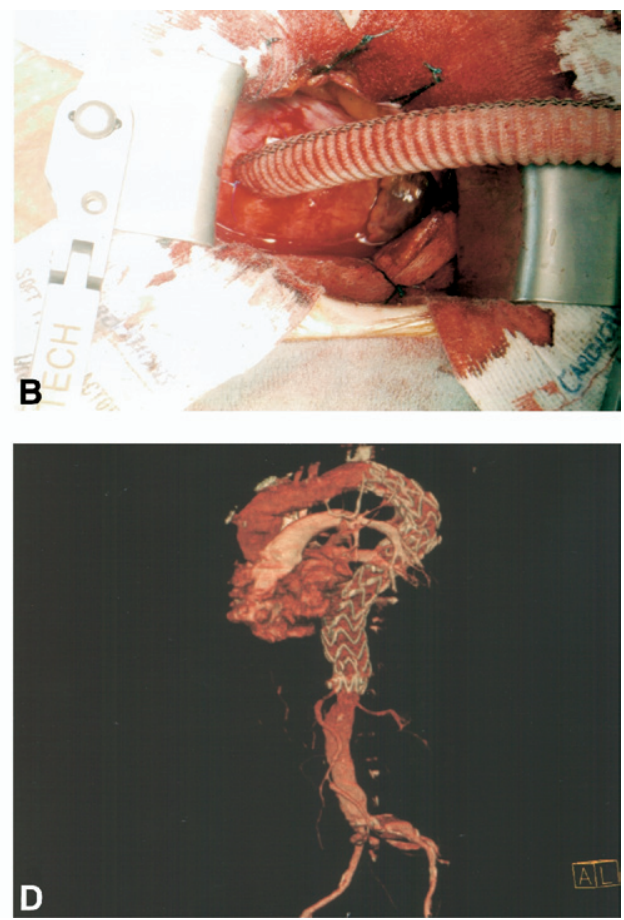

Figure 1. A CT angiogram depicts aortic aneurysm involving the descending aorta $(A)$ and the result of stent grafting of the descending aorta (D). A 10-mm Dacron T-tube was sutured in the ascending aorta (B). A 24F introducer was placed into the Dacron graft and hemostasis was achieved with a standard snare technique (C).

clamp the ascending aorta without video assistance. A nondiseased segment of aorta was identified by direct palpation and arterial pressure was lowered to minimize aortic tension during crossclamping. After the administration of heparin $(100 \mathrm{U} / \mathrm{kg}$ with a target activated clotting time of about 250 seconds), a $10-\mathrm{mm}$ Dacron T-tube was sutured to the lateral side of the ascending aorta, $3 \mathrm{~cm}$ below the origin of brachiocephalic trunk, under direct vision and driven out through the thoracotomy (Figure 1, B). A $45^{\circ}$ angled aorta-graft anastomosis was performed to favor right advancement of the stent grafts toward the aortic arch, and the second intercostal space was chosen to optimize the direction of the Dacron tube. A $24 \mathrm{~F}$ introducer was placed into the Dacron graft and hemostasis was achieved with a standard snare technique (Figure 1,C). From a 4F left brachial approach, a pigtail catheter (Cordis; Johnson \& Johnson, Warren, NJ) was positioned in the ascending aorta to assess the correct position of the proximal margin of the LSA. A superstiff guide wire (Back-Up Meier, Boston Scientific, Nanterre, France) and pigtail catheter were inserted within the introducer followed by two Valiant (Medtronic, Inc, Minneapolis, Minn) stent grafts to exclude the aneurysm between the LSA and the superior mesenteric artery (SMA) (Figure $1, D)$. The LSA was not excluded. Inasmuch as the safe distal landing zone was closed by the SMA, the celiac trunk was previously coiled by means of an Amplatzer device (AGA Medical Corporation, Plymouth, Minn). A compliance balloon occlusive test of the celiac trunk by contemporary contrast media injection in the SMA was performed before the procedure to confirm the

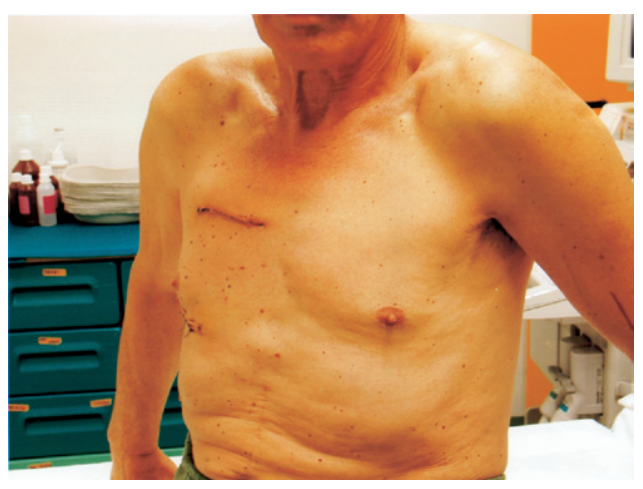

Figure 2. A 7.0-cm incision was made at the level of the second right anterior intercostal space.

presence of collateral blood supply. A transesophageal echocardiogram and an intraoperative angiogram confirmed the absence of any endoleaks. The Dacron graft was then sutured close to the aorta.

The patient was extubated after 4 hours in the intensive care unit and transferred to the ward on the first postoperative day. On the second day he started walking and was able to walk on his own. One-, 6-, and 12-month CT angiograms showed excellent recon- 
struction with complete exclusion of the aneurysm and absence of endoleaks.

A residual subrenal abdominal aortic aneurysm has been scheduled for surgical treatment.

\section{Discussion}

Currently, early and midterm results of endovascular treatment of descending thoracic aneurysms are encouraging and offer an alternative option for patients unsuitable for conventional surgery. The procedure is associated with significant reduction of perioperative mortality, stroke, and paraplegia, particularly in high-risk patients. $^{2}$

Traditionally, the common femoral artery remains the first choice for access with the iliac artery, abdominal aorta, and right subclavian artery being the second choice. If all these sites are diseased, the ascending aorta becomes the last option, as occurred in our patient. Furthermore, although the right subclavian artery is usually a good vessel for size and wall quality, it often presents an incorrect angle with the brachiocephalic trunk and aortic arch. Consequently, insertion of an introducer could become a high-risk procedure.

The purpose of our method is to minimize procedural risks, with less morbidity and faster recovery. Use of a right anterior minithoracotomy and side-bite clamping allows a 10-mm Dacron graft to be easily anastomosed to the ascending aorta, without sternotomy, ${ }^{3}$ cardiopulmonary bypass, ${ }^{4}$ or video assistance. ${ }^{5}$ In particular, video assistance is not required, as opposed to the technique described by Bernier and coworkers, ${ }^{5}$ and the consequent learning curve can thereby be avoided.

This approach offers the possibility to treat the entire descending thoracic aorta and not only focal lesions localized in the proximal stretch through a left minithoracotomy, as described by Bernier and associates, ${ }^{5}$ who used a Dacron graft sutured to the descending aorta distal to the lesion. Furthermore, the surgeon is able to recognize the best site on the ascending aorta to perform side-bite clamping and to direct explore the aortic wall by touching it or by epicardial echocardiography.

In conclusion, this novel approach to treat thoracoabdominal aortic aneurysms seems to be safe and efficient, with minor discomfort for the patient and requiring a lower grade of general expertise than that required in the case of challenging aortic disease.

\section{References}

1. Zipfel B, Hammerschmidt R, Krabatsch T, Buz S, Weng Y, Hetzer R. Stent-grafting of the thoracic aorta by the cardiothoracic surgeon. Ann Thorac Surg. 2007;83:441-9.

2. Bavaria JE, Appoo JJ, Makaroun MS, Verter J, Yu ZF, Mitchell RS. Endovascular stent grafting versus open surgical repair of descending thoracic aortic aneurysm in low-risk patients: a multicenter comparative trial. J Thorac Cardiovasc Surg. 2007;133:369-77.

3. Dambrin C, Marcheix B, Hollington L, Rousseau H. Surgical treatment of an aortic arch aneurysm without cardio-pulmonary bypass: endovascular stent-grafting after extra-anatomic bypass of supra-aortic vessels. Eur J Cardiothorac Surg. 2005;27:159-61.

4. Usui A, Tajma K, Nishikimi N, Ishiguchi T. Implantation of an endovascular covered stent-graft for distal aortic arch aneurysm via midsternotomy under pigtail catheter guidance. Eur J Cardiothorac Surg. 1999;16:356-8.

5. Bernier PL, Turcotte R, Normand JP, Dagenais F. Video-assisted mini-thoracotomy for thoracic stent-graft implantation: a novel vascular access for endovascular repair. $J$ Endovasc Ther. 2004;11: $180-2$. 